\title{
14 \\ Concept of Quantified Abstract Quotient Automaton and its advantage
}

\author{
G. JUANOLE and L. GALLON \\ $L A A S-C N R S$ \\ 7, avenue du colonel Roche \\ 31077 Toulouse cedex 4 (France) \\ E-mail : \{juanole, gallon\}@laas.fr
}

\begin{abstract}
At first, we define the concept of quantified abstract quotient automaton (which concerns state graphs with qualitative and quantitative labels) and present a methodology for computing it. It is an abstract view which results, on the one hand, of the quotient automaton obtained according to an equivalence relation based on qualitative labels, and, on the other hand, of reduction rules applied to the quantitative labels. This abstract view has the nice property of offering both verification and evaluation capacities (interesting point for the analysis of Qualities of Service, for example). The advantage of this concept for the bottom-up modeling and analysis of multi-layer communication architectures is then demonstrated.
\end{abstract}

\section{Keywords}

Transition systems, Petri nets, qualitative and quantitative labels, verification, quality of service, evaluation, communication networks, real-time systems

\section{INTRODUCTION}

- The labeled transition systems (or still labeled state graphs) are formalisms extensively used for representing the dynamic behavior of distributed systems (set of communicating entities). A labeled state graph consists of a set of global states, a set of arcs linking the states and a set of labels associated 
with the set of the arcs. More often the labels are of the qualitative type appearing in the form of Predicate/Action (a Predicate can be a message reception; an Action can be a message emission). A labeled state graph allows, in particular, the analysis of specific properties of the system being modeled (properties which depend on the system mission and which can be analyzed through the interpretation of transition sequences and the associated labels).

- In many cases the observation that we want to do on a labeled state graph only concerns a few events (events which are relevant from a point of view) and then it is important, in order to facilitate the analysis, to get a reduced labeled state graph which is equivalent to the initial labeled state graph according to an equivalence relation (the reduced labeled state graph is still called the quotient automaton). The observational equivalence relation [Mil80] is a very powerful equivalence relation which has been largely used in the field of the communication protocol verification (verification relative to the service supplied [FJV90]).

- However today, in particular, in the context of real-time systems, we cannot be satisfied with the representation of the dynamic behavior of the systems with state graphs which only have qualitative labels on the transitions. We have to use state graphs with both qualitative and quantitative labels (durations and/or probabilities). In this context, the observational equivalence relation is still relevant (we want still to observe a system from the point of view of a few events) but we want too (in order to be able to evaluate the performances related to these events) to add to the quotient automaton quantitative informations (which depend, of course, on the quantitative labels of the initial labeled state graph).

The quantification of the quotient automaton offers an object which allows to merge verification and evaluation activities. In our knowledge, there were never works on the quantification of quotient automata. That is the goal of this paper. However we want to say that this study does not give new theory. It proposes to combine (that is new) already existing theoretical results (observational equivalence [Mil80]; quantitative abstract views [AJ95]) for the definition of the concept of Quantified Abstract Quotient Automaton and to show its advantage for the modeling and analysis in communication architectures.

This paper is divided into three sections. In the first section, we present the background which is necessary. In the second section, we present a methodology for computing a quantified abstract quotient automaton and illustrate it through an example of a service provided by a protocol. In the third section, by considering a two layer communication architecture, we show the advantage of the quantified abstract quotient automaton concept for evaluating qualities of service and controlling models sizes. 


\section{BACKGROUND}

\subsection{State graph with qualitative labels}

(a) Definition

A finite state graph labeled with qualitative labels is a triplet $\langle S, E, \Delta\rangle$ where :

- $S=\left(S_{0}, S_{1}, \ldots\right)$ is the set of the vertices of the graph; they represent the states of the system being represented; $S_{0}$ is the initial state,

- $E=\left(e_{0}, e_{1}, \ldots\right)$ is the set of labels (events),

- $\Delta$ is the set of the labeled arcs of the graph (i.e. $\Delta \subset S \times E \times S$ ); an element $(m, \mu, p) \in \Delta$ is denoted $m \stackrel{\mu}{\rightarrow} p$. $\stackrel{\mu}{\rightarrow}$ is a transition relation.

An example of such graph is represented on figure 1.

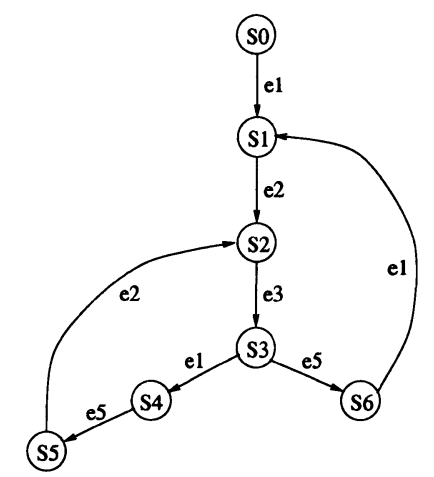

Figure 1 State graph with qualitative labels

\section{(b) Observational equivalence relation and quotient}

\section{automaton}

Call $E^{\prime}$ the subset of the events of a state graph with qualitative labels that we want to observe (observable or visible events) and call $\tau$ an event which represents any event that we do not want to observe ( $\tau$ is called an unobservable or invisible or internal event).

A transition relation $\left\{\stackrel{\mu}{\Rightarrow}, \mu \in E^{\prime} \cup\{\epsilon\}\right\}$ is defined in a standard way by :

- $m \stackrel{\epsilon}{\Rightarrow} p: m=p$ or $m \stackrel{\tau}{\rightarrow} x_{1} \stackrel{\tau}{\rightarrow} \ldots \stackrel{\tau}{\rightarrow} x_{p} \stackrel{\tau}{\rightarrow} p$

- $m \stackrel{\mu}{\Rightarrow} p: m \stackrel{\epsilon}{\Rightarrow} x_{1} \stackrel{\mu}{\rightarrow} x_{2} \stackrel{\epsilon}{\Rightarrow} p$ 


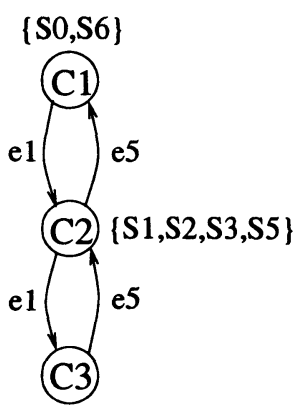

\{S4\}

Figure 2 Quotient automaton

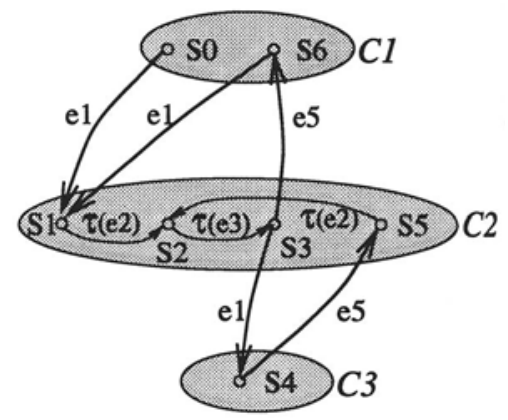

Figure 3 Detailed quotient automaton

Such relation represents what it is still called either $\epsilon$-experimentation or $\mu$ experimentation.

Now, we can define the observational equivalence relation (noted $\equiv$ ) which can be seen as a decreasing sequence of equivalence relations. This relation can be defined by recurrence [Ver89] :

- $\forall m, p \in S, m \equiv_{0} p$

- $m \equiv_{k+1} p$ if and only if $m \equiv_{k} p$ and $\forall t \in E^{\prime} \cup\{\epsilon\}$,

$$
\begin{aligned}
& {\left[m \stackrel{t}{\Rightarrow} n \text { implies } \exists q \in S: p \stackrel{t}{\Rightarrow} q \text { and } n \equiv_{k} q\right]} \\
& {\left[p \stackrel{t}{\Rightarrow} q \text { implies } \exists n \in S: m \stackrel{t}{\Rightarrow} n \text { and } q \equiv_{k} n\right]}
\end{aligned}
$$

Informally, two states are observationally equivalent if the observed behaviors of the system being modeled from these states are similar : two states are equivalent at the order $\mathbf{k}$ if for any $\mathrm{t}$-experimentation from one state, we have a t-experimentation from the other state which is equivalent at the order (k-1).

If we come back to the state graph of the figure 1 and if we consider the events $e_{1}$ and $e_{5}$ as the only observable events, we get the quotient automaton represented on the figure 2 (this quotient automaton has three state classes $C_{1}, C_{2}$ and $C_{3}$ ).

The decreasing sequence of the equivalence relations is :

$$
\begin{aligned}
& \equiv_{0}=\left\{S_{0}, S_{1}, S_{2}, S_{3}, S_{4}, S_{5}, S_{6}\right\} \\
& \equiv_{1}=\left\{\left\{S_{0}, S_{6}\right\},\left\{S_{1}, S_{2}, S_{3}, S_{4}, S_{5}\right\}\right\} \\
& \equiv_{2}=\left\{\left\{S_{0}, S_{6}\right\},\left\{S_{1}, S_{2}, S_{3}, S_{5}\right\},\left\{S_{4}\right\}\right\}
\end{aligned}
$$

\section{(c) Detailled quotient automaton}

In the quotient automaton, we do not worry about the links between the states in a state class (the information on these links is not relevant from the 


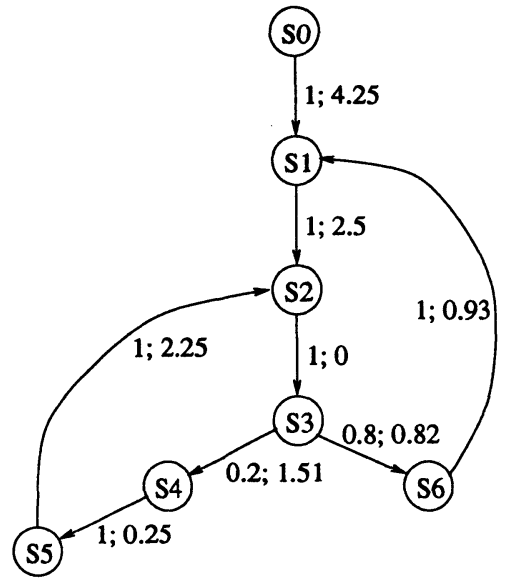

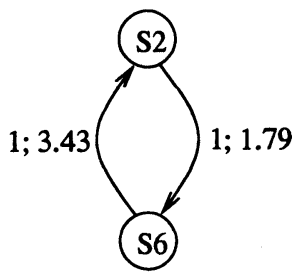

Figure 5 abstract view

Figure 4 state graph with quantitative labels

strict point of view of the observational equivalence relation). We propose to call detailed quotient automaton the quotient automaton where we have the representation of the links between the states of each state class (the detailed quotient automaton is immediately got, on the one hand, from the classes of the quotient automaton and, on the other hand, by looking at the initial state graph in order to have the links between the states in each state class).

The detailed quotient automaton of the state graph of the figure 1 is represented on the figure 3 . Note that here (in the considered example) the transitions between the classes only result of the observable events (but, in a general case, event $\tau$ can also induce transitions between classes).

\subsection{State graph with quantitative labels}

\section{(a) Definition}

Graphs $G$ that we consider are constituted of a set $S$ of states and a set $U$ of arcs connecting the states. Let an arc $u_{i j}$ connecting a state $S_{i}$ to a state $S_{j}$; the arc $u_{i j}$ is characterized by a doublet : $p_{i j}$ (transition probability), $\theta_{i j}$ (transition duration). An example is presented on the figure 4 . Note that we only consider here graphs of the "1-graph" type i.e. graphs for which there never exists more than one arc between any two states $S_{i}$ and $S_{j}$ taken in this order.

In order to formally specify the quantitative informations, two types of square matrices of order equal to the cardinal of the set of the states (let $Z$ this cardinal) are associated to the graph ([AJ95]) : 
a matrix $\mathcal{P}$ of direct transition probabilities between states : $\mathcal{P}=\left[p_{i j}\right]$ with

$$
\forall i=1,2, \ldots, Z \text { and } \forall j=1,2, \ldots, Z \text { we have } 0 \leq p_{i j} \leq 1 \text { and } \sum_{j=1}^{Z} p_{i j}=1
$$

- a matrix $\Theta$ of the durations of the transitions between states : $\Theta=\left[\theta_{i j}\right]$.

Note that when the graph is not complete, i.e. with probabilities $p_{i j}$ equaling zero between state pairs $\left(S_{i}, S_{j}\right)$, it can be stated that there exists between these state pairs, fictitious transitions characterized by infinite durations.

\section{(b) Abstract view}

An abstract view of a graph $G$ is a reduced graph obtained from a projection on a subset of states from graph $G$ (observed graph) : the world of observed states is equivalent to the sub-world of these states in the context of the graph $G$ on the basis of the so-called notion of "first passage" ([AJ95]).

Let an arc $u_{k l}$ connecting a state $S_{k}$ to a state $S_{l}$ of the reduced graph. The arc $u_{k l}$ is also characterized by a doublet : $F_{k l}$ (transition probability at the first passage between states $S_{k}$ and $S_{l}$ ), $T_{k l}$ (mean duration at the first passage between states $S_{k}$ and $\left.S_{l}\right) . F_{k l}\left(T_{k l}\right)$ depends on $p_{k l}\left(\theta_{k l}\right)$ but also on the probabilities and the durations between the states of the graph $G$ which are not observed and which are on the paths between the states $S_{k}$ and $S_{l}$. General formulas for obtaining $F_{k l}$ and $T_{k l}$ from the matrices $\mathcal{P}$ and $\Theta$, and which are a generalization of the Beizer's reduction rules and the Chapman-Kolmogorov formulas [How71], are given in [AJ95].

For example, if we consider the graph of the figure 4 , and by making a projection on the states $S_{2}$ and $S_{6}$, we get the abstract view of the figure 5 .

\subsection{State graph with qualitative and quantitative labels}

A state graph with qualitative and quantitative labels is a state graph where each arc is labeled with a triplet (event, probability, duration). If we consider the graph of the figure 1 and we add to the arcs the labels of the graph of the figure 2, we get such a graph. Such a graph is an interesting structure, specially in the context of real-time systems, to evaluate Real-Time Computation Tree Logic [AES89] and/or Probabilistic Real-Time CTL [HJ91] formulas.

Generally, state graphs (with or without qualitative and/or quantitative labels) are not the first specification of a system, but represent the dynamic behavior of a higher level model like Petri nets, for example. We present now the Petri net based model that we use (Stochastics Timed Petri Nets (STPN) [JA91, JG95]). 


\subsection{Stochastic Timed Petri Nets (STPN)}

\section{(a) Definition}

An STPN is a Petri net where a probability density function is associated to each transition. A density probability function can be a continuous distribution (exponential, uniform), a deterministic distribution (duration $\neq 0$ or 0 ), a mixed distribution (uniform and discrete). Transitions with a duration equal to 0 are called immediate transitions (all the other transitions are called timed transitions). The selection of the transition to be fired [ $\mathrm{MBB}^{+}{ }^{85}$ ] is based, in the general case, on the race model and, in some particular cases (transitions, with identical deterministic distributions, which are simultaneously enabled) on the preselection model with an equiprobable choice. The conditioning on the past history is the enabling memory $\left[\mathrm{MBB}^{+} 85\right]$.

The dynamic behavior is represented by a randomized state graph which is a state graph with qualitative and quantitative labels : a qualitative label is made of the name of the transition of the underlying Petri net which has induced a state change, and the name of the events associated to this transition; the quantitative label associated to a qualitative label is the doublet probability-duration of the transition firing.

\section{(b) Example}

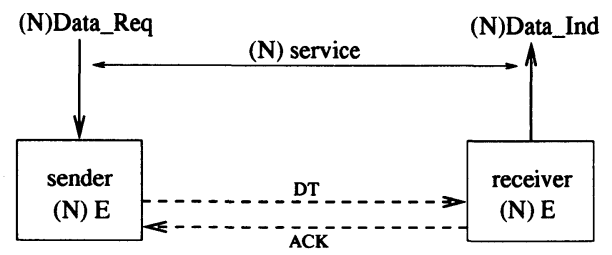

Figure $6(\mathrm{~N})$ layer

- Consider an (N) layer of a communication architecture (figure 6) which provides an unidirectional data transfer (N)service (the (N)primitives are (N)Data_ Req and (N)Data_Ind) and which is based on a "stop and wait" protocol [BSW69] (the sender (N)E sends the (N)PDU DT and waits for the (N)PDU ACK sent by the receiver $(\mathrm{N}) \mathrm{E}$; the (N)PDU losses are controlled by a time-out mechanism in the sender (N)E). Furthermore, concerning the ACK loss, in order to have not to consider a numbering scheme and then to have a simplest model, we suppose an intelligent medium in the direction receiver (N)E - sender (N)E : when there is an ACK loss, this medium signals the loss to the receiver which records this fact (then when the next DT (duplicated DT) will be received, the receiver (N)E will discard it and will send again $\mathrm{ACK}$ ). 


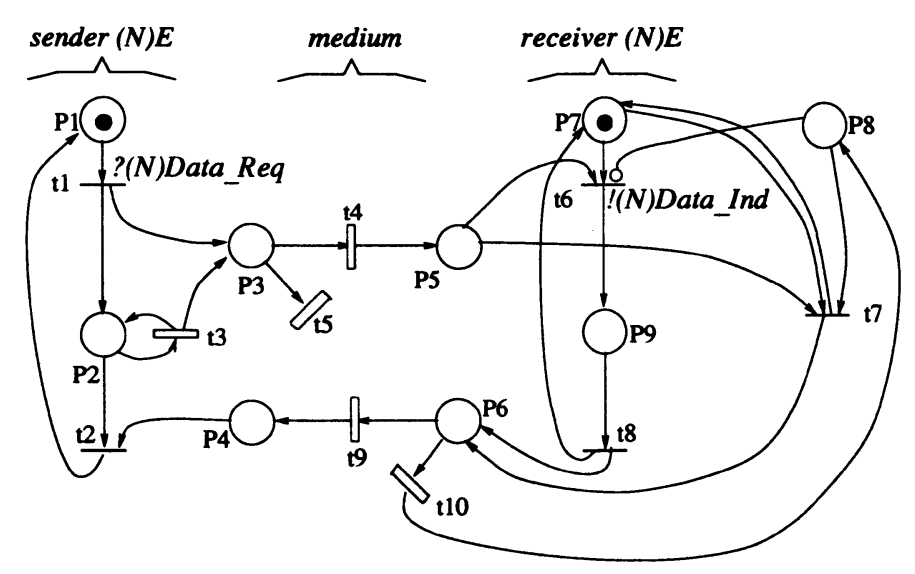

Figure 7 STPN model

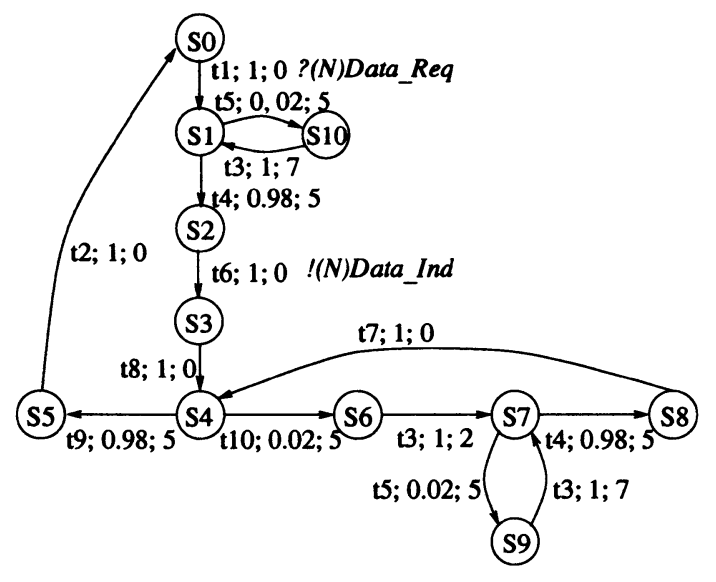

Figure 8 Randomized state graph

- The STPN model is represented on the figure 7 (immediate transitions are represented with a thin line; timed transitions are represented with a rectangle). Transitions $t_{1}$ and $t_{6}$ are respectively labeled with the (N)Data_Req reception (noted ?(N) Data_Req) and the (N)Data_Ind emission (noted !(N)Data_Ind). Transitions $t_{4}$ and $t_{9}$ represent the transmission of respectively DT and ACK (note that after an ACK loss, we put a token in place $P_{8}$ which records the fact "ACK loss"). The time-out and the retransmission are represented by the transition $t_{3}$.

The choice of the distribution associated to the loss transitions $\left(t_{5}, t_{10}\right)$ with respect to the transmission transitions $\left(t_{4}, t_{9}\right)$ allow to simulate loss probabilities (general formulas are given in [JA91]).

- The randomized state graph (by considering the loss probability of $2.10^{-2}$ ) is represented on the figure 8 (concerning the qualitative labels : we repre- 
sent all the names of the transitions of the underlying Petri net, and only the events related to the $(\mathrm{N})$ service).

\section{QUANTIFIED ABSTRACT QUOTIENT AUTOMATON}

\subsection{Methodology for obtaining it}

We have three steps.

The first step consists in obtaining the quotient automaton according to the observational equivalence relation (i.e. by only considering the qualitative labels and by observing a subset of events); the quotient automaton only provides the classes (with the states included in each class) and the transitions between the classes with their labels (visible or invisible event).

The second step consists in obtaining the quantified detailed quotient automaton i.e. we make to appear the arcs between the states in each state class (as shown in the subsection 2.1.3) and we associate to each arc (between the state classes and between the states in each state class) a probability and a duration (this quantification is immediate from the initial state graph with qualitative and quantitative labels). At this step, each class is represented by a quantified detailed view.

The third step consists in obtaining, for each class, a quantified abstract view i.e. we evaluate the quantified behavior as seen by its environment (the adjacent classes). This is the important point of the quantified abstract quotient automaton concept. We present it now.

\subsection{Quantified abstract view of a state class}

Consider a quantified detailed view of a state class $C$ (figure 9 ). In the class, each transition (its qualitative labels (the invisible event $\tau$ ) has not been represented here) has the quantitative labels $p_{x}$ (probability) and $\theta_{x}$ (duration). Note that concerning the transitions coming and/or going to the others state classes, we only have represented here the qualitative labels (visible events $e_{i}$ and invisible event $\tau$ ).

We define two types of states : states which are at the frontier of the class with others classes and that we call frontier states (the states $S_{i}, S_{j}, S_{k}, S_{l}$ are the frontier states of the class $C$ ), and the internal states which represent the behavior inside a class (the states $S_{m}, S_{n}, S_{o}$ are the internal states of the class $C$ ).

Among the frontier states, we have input states (they represent entries in the class; the state $S_{i}$ is an input state), output states (they represent exits from the class; the state $S_{l}$ is an output state) and input/output states (they represent both entries and exits from the class; states $S_{j}$ and $S_{k}$ are input/output states). 


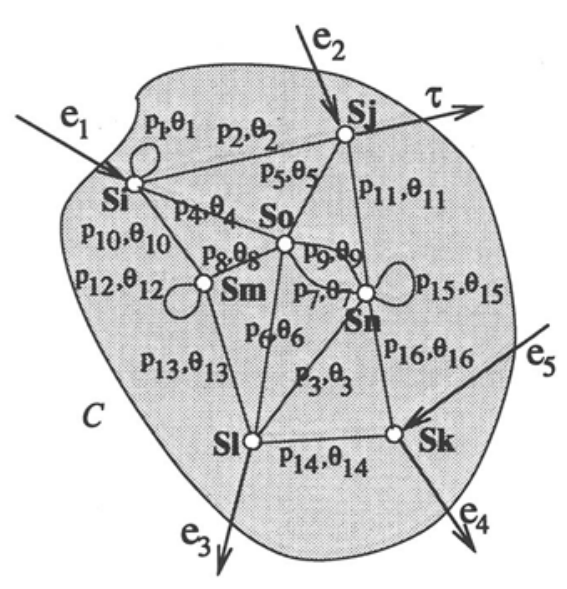

Figure 9 Quantified detailed view of a class

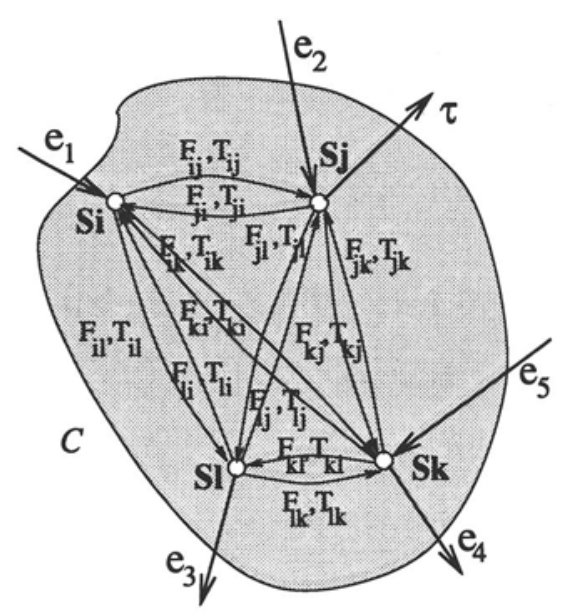

Figure 10 Quantified abstract view of a class

We can have loops on each type of state.

\section{Definition :}

A quantified abstract view of a class models the quantitative behavior of a class between the frontier states, i.e it is represented by a graph with quantitative labels which only has the frontier states as vertices and which is irreflexive (no loops).

The quantified abstract view of the quantified detailed view of the figure 9 is represented on the figure 10. Each transition between an input frontier state $S_{a}$ and an output frontier $S_{b}$ is labeled with a probability $F_{a b}$ and a duration $T_{a b}$ which are respectively the probability and the mean duration of the transition between the states $S_{a}$ and $S_{b}$ on the basis of the so-called notion of "first passage" [How71] and knowing that since the departure instant from the frontier state $S_{a}$ no other frontier state (except $S_{a}$ if we have loops around it and/or circuits through internal states which come back to $S_{a}$ ) is traversed. The general formulas for computing $F_{a b}$ and $T_{a b}$ are easily obtained from the works presented in [AJ95] on the quantitative abstract views.

\section{Remarks:}

1. the reason of considering an irreflexive graph is that we want to represent the behavior of a class as strictly seen by the environment of the other classes (so loops, if any, need not to appear explicitly);

2. from a probabilistic point of view, a loop around a state $S_{a}$ can always be removed by integrating its probability in the outgoing arcs probabilities;

3. if a quantified detailed view of a class has only frontier states and no loop, we have already its abstract view and we do not need obviously any computation. 


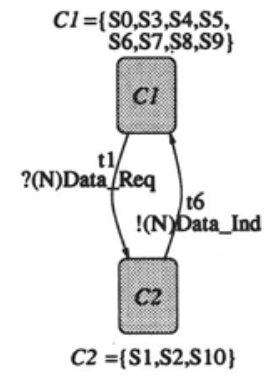

Figure 11 Quotient automaton

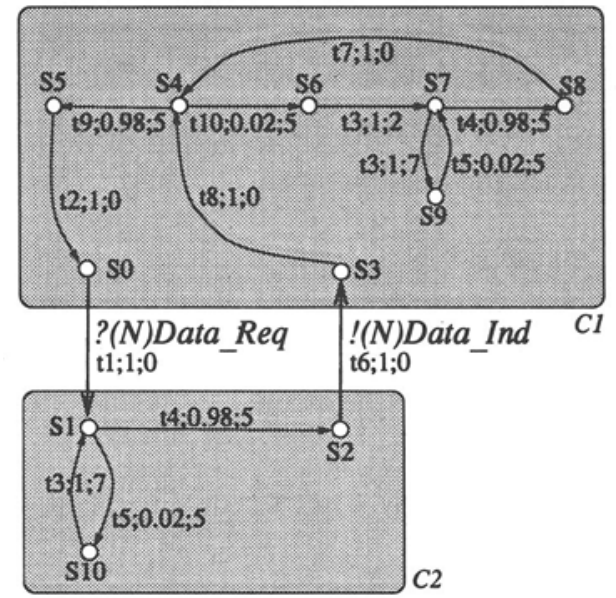

Figure 12 Quantified detailed quotient automaton

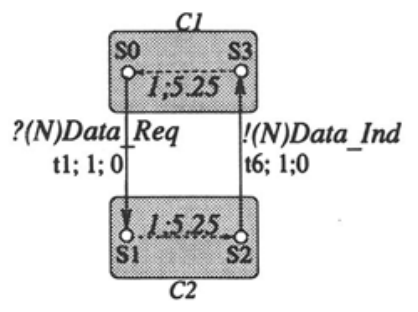

Figure 13 Quantified abstract quotient automaton

\subsection{Example}

Consider again the randomized state graph of the figure 8 (behavior of an (N) layer of a communication architecture) and suppose that we only are interested in the $(\mathrm{N})$ service view. So we apply the quantified abstract quotient automaton concept (events?(N)Data_Req and !(N)Data_Ind are the observed events).

Figures 11,12 and 13 represent the three steps to obtain the quantified abstract quotient automaton. The figure 11 gives the quotient automaton (we have two state classes $C_{1}$ and $C_{2}$ (with the list of their states) which are linked with two arcs labeled with the name of the Petri net transitions and with ?(N)Data_Req and !(N)Data_Ind). The figure 12 gives the quantified detailed quotient automaton (this view combines the knowledge of the quotient automaton (figure 11) and the randomized state graph (figure 8)); the figure 13 gives the quantified abstract quotient automaton (each quantified detailed 


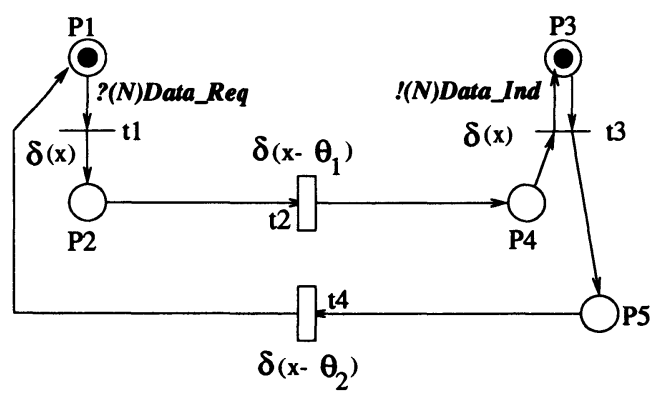

Figure 14 STPN model

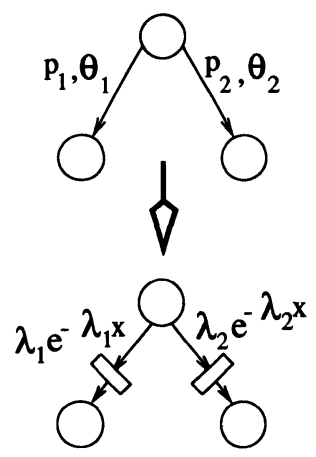

Figure 15 choice translation

class has been replaced by a quantified abstract class) i.e. we have the view which is relevant for an user of the (N) layer both in terms of qualitative and quantitative informations. Note that if we make changes in the loss probability values, the structure of the quantified abstract quotient automaton does not change (only the sojourn times in the classes $C_{1}$ and $C_{2}$ change).

\subsection{Comments}

We want to emphasize three points about the quantified abstract quotient automaton :

1. it allows to quantify the divergence phenomenon [Hen88] which can occur in a quotient automaton (if we have loops and/or circuits in a state class, we can then, from a qualitative point of view, stay endless in the class). The consideration of the quantitative information gives a realistic view;

2 . it is a good concept to characterize both qualitatively and quantitatively Qualities of Service (QoS). For example, in the previous example, we can say :

- from a qualitative point of view (the information was already in the quotient automaton), the (N) service is inevitably provided (sequence ?(N)Data_Req, !(N)Data_Ind);

- from a quantitative point of view :

- the time to provide the (N) service (i.e. from ?(N)Data_Req to !(N)Data_Ind) is $\theta_{1}$;

- the period of the (N) service (i.e. from a ?(N)Data_Req to a new ?(N)Data_Req) is $\theta_{1}+\theta_{2}$.

3. from the quantified abstract quotient automaton we can directly extract a stochastic timed Petri net model which can be used, for modeling the $(\mathrm{N}+1)$ layer (bottom-up modeling). For example, if we come back to the quantified abstract quotient automaton of the figure 13, we can translate it into the STPN model of the figure 14 (the places $P_{4}$ and $P_{5}$ and the 


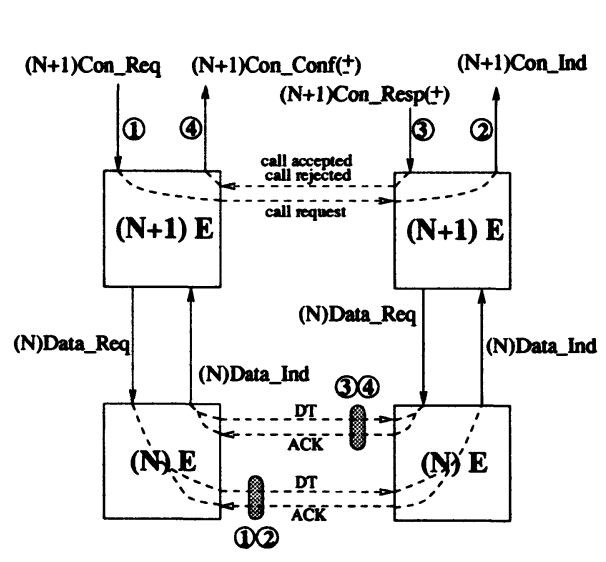

Figure 16 Two layer architecture

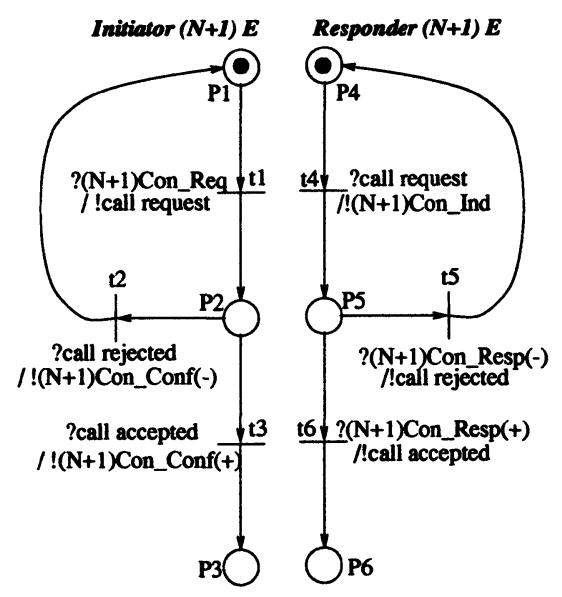

Figure $17(\mathrm{~N}+1)$ entities

timed transition $t_{2}$ with the deterministic distribution $\delta\left(x-\theta_{1}\right)$ represent the class $C_{1}$; the places $P_{5}$ and $P_{1}$ and the timed transition $t_{4}$ with the deterministic distribution $\delta\left(x-\theta_{2}\right)$ represent the class $C_{2}$; the place $P_{1}$ represents the sender (N)E ready to receive an (N)Data_Req; the place $P_{2}$ represents the receiver $(\mathrm{N}) \mathrm{E}$ ready to send (N)Data_ind; the transitions labeled with ?(N)Data_Req and !(N)Data_Ind are immediate $(\delta(x))$.

This example is simple in the sense that the automaton has no choice. But in the general case, we can have choices which will induce also Petri nets with choices (transitions in competition). Two sub-cases must be considered according to the values of the unconditional sojourn times in the states, from where there is a choice, are 0 or different from 0 :

- if 0 , each transition in competition will be an immediate transition (i.e. a deterministic distribution $\delta(x)$ ) and will have a probability which is the probability of the corresponding transition in the automaton;

- if different from 0, each transition in competition will be a timed transition with an exponential distribution as it is represented on the figure 15 . The exponential distributions are characterized by what are called equivalent rates [Flo85]: $\lambda_{1}=\frac{p_{1}}{p_{1} \theta_{1}+p_{2} \theta_{2}} ; \lambda_{2}=\frac{p_{2}}{p_{1} \theta_{1}+p_{2} \theta_{2}}$.

\section{ON THE ADVANTAGE OF THE QUANTIFIED ABSTRACT QUOTIENT AUTOMATON CONCEPT}

Consider the two layer communication architecture represented on the figure 16 where the $(\mathrm{N}+1)$ layer must open a $(\mathrm{N}+1)$ connection by using a data transfer $(\mathrm{N})$ service which is based on a fragmentation process $((\mathrm{N})$ SDU fragmentation) and a "stop and wait" protocol as the one presented in the 


\begin{tabular}{|c|c|c|c|c|}
\hline number of fragments & 2 & 3 & 4 & 5 \\
\hline number of states & 24 & 35 & 46 & 57 \\
\hline
\end{tabular}

Table 1 unidirectional data transfer (N) service

\begin{tabular}{|c|c|c|c|c|c|c|}
\hline \multicolumn{2}{|c|}{ number of fragments } & 2 & 3 & 4 & 5 & \\
\hline only & brute force & 136 & 192 & 243 & 293 & \multirow{4}{*}{$\begin{array}{c}\text { number of } \\
\text { states }\end{array}$} \\
\hline Resp + & QAQA concept & \multicolumn{4}{|c|}{15} & \\
\hline \multirow{2}{*}{$\begin{array}{c}\text { Resp + } \\
\text { and - }\end{array}$} & brute force & 698 & 931 & 1139 & 1374 & \\
\hline & QAQA concept & \multicolumn{4}{|c|}{26} & \\
\hline
\end{tabular}

Table $2(\mathrm{~N}+1)$ service (connection opening)

subsection 2.4.(b) We suppose that, like in the subsection 2.4.(b), we can have (N) PDU losses.

If we want to analyze and evaluate the $(\mathrm{N}+1)$ connection opening, we must model this communication architecture. This study can be made in two different ways :

1. either we adopt a "brute force" technique, i.e. we make a global modeling by interconnecting the STPN models of the $(\mathrm{N}+1)$ entities (figure 17) and the data transfer (N) service in each direction (such a model is easily obtained from the model given on the figure 7 by integrating the fragmentation (we do not represent it here for space reasons);

2. or we are more artful, i.e. we proceed in two steps :

- we model the data transfer (N) service in each direction (the table 1 gives the number of states as a function of the number of fragments for sending a (N) SDU) and we compute the quantified abstract quotient automaton from which we extract an STPN model (their structures are independent of the fragmentation process; we have structures identical to the one presented on the figures 13 and 14, only the values of $\theta_{1}$ and $\theta_{2}$ change because of the fragmentation);

- we make a global model by interconnecting the models of the $(\mathrm{N}+1)$ entities with the STPN models got from the quantified abstract quotient automaton (QAQA);

The table 2 summarizes the advantage of the QAQA concept to make bottom-up modeling and to master the size of the models.

Remarks :

1. When we only make a qualitative analysis, it is well known that the quotient automaton has this advantage. The quantified abstract quotient automaton extend this advantage to the quantitative area;

2. We could obviously evaluate the QoS of the $(N+1)$ connection opening (duration) but we only wanted here to emphasize the advantage of the quantified abstract quotient automaton concept for controlling the models size; 
3. We could also obviously obtain the quantified abstract quotient automaton concerning the $(\mathrm{N}+1)$ service and extract a STPN model in order to continue the bottom-up modeling.

\section{CONCLUSION}

The concept of quantified abstract quotient automaton that we have defined in this paper provides abstract views of the behavior of a system both in terms of events and quantitative informations related to these events. It is a powerful concept to study real-time distributed systems (systems growing intensively today in many areas) which require qualitative and quantitative analysis. In particular, we can characterize Qualities of Service and implement bottom-up modeling techniques which are very important with multi-layer systems in order to control the size of the models. These points have been shown on a simple example of a communication architecture (note that the sophisticated data transfer protocols with go-back $\mathrm{n}$ or selective reject procedures have been analysed in our laboratory works).

This concept has been implemented in the context of a formal modeling method based on the "Stochastic Timed Petri net" model which is developed in our laboratory [JA91, JG95].

\section{REFERENCES}

[AES89] A. Emerson, A. Mok A. Sistla and J. Srinivasan. Quantitative temporal reasoning. In Workshop on Automatic Verification Methods for Finite States Systems, Grenoble, France, 1989.

[AJ95] Y. Atamna and G. Juanole. Methodology for Obtaining Abstract Views of State Graphs Labeled with Probabilities and Times : an Example of Application to a Communication Protocol. In MASCOTS'95, the Third International Workshop on Modeling, Analysis and Simulation of Computer and Telecommunication Systems, pages 299-306, Durham, North Carolina, january 1995.

[BSW69] K.A. Bartlett, R.A. Scantlebury, and P.T. Wilkinson. A note on realible full-duplex transmission over half-duplex links. In Commun. Ass. Comput. Mach., volume 12. may 1969.

[FJV90] C. Faure, G. Juanole, and F. Vernadat. LAPD Protocol at the ISDN Interface : Formal Modelling with Petri Nets Based Models and Verification by Abstraction (String, Observational and Behavioral Equivalences). In $10^{\text {th }}$ International Conference on Computer Communication, New Dehli, India, november 1990.

[Flo85] G Florin. Réseaux de Petri Stochastiques : théorie et techniques de calcul. PhD thesis, Université Pierre et Marie Curie (Paris 
VI), 1985.

[Hen88] M. Hennessy. Algebric Theory of Processes. The MIT Press, 1988.

[HJ91] H. Hansson and B. Jonsson. A framework for reasoning about time and reliability. In Tenth IEEE Real-Time Systems Symposium, 1991.

[How71] R.A. Howard. Dynamic Probabilistic Systems, volume 2 : SemiMarkov and Decision Process. Wiley, J and Sons, INC., 1971.

[JA91] G. Juanole and Y. Atamna. Dealing with Arbitrary Time Distributions with the Stochastic Timed Petri Net Model. Application to Queueing Systems. In PNPM'91, the Fourth International Workshop on Petri Nets and Performance Models, pages 32-43, Melbourne, Australia, December 1991.

[JG95] G. Juanole and L. Gallon. Critical Time Distributed Systems : Qualitative and Quantitative Analysis based on Stochastic Timed Petri Nets. In FORTE'95, the $8^{\text {th }}$ International IFIP Conference on Formal Description Techniques for Distributed Systems and Communication Protocols, Montreal, Canada, october 1995.

[MBB $\left.{ }^{+} 85\right]$ A.M. Marsan, G. Balbo, A. Bobbio, G. Conte, G. Chiola, and A. Cumani. On Petri Nets with Stochastic Timing. In TPN'85, the First International Workshop on Time Petri Nets, Torino, Italia, 1985.

[Mil80] R. Milner. A Calculus of Communication Systems, volume 92. Springer Verlag, Berlin Heidelberg, 1980.

[Ver89] F. Vernadat. Vérification Formelle d'Applications Réparties. Caractérisation Logique d'une Equivalence de Comportement. PhD thesis, Université Paul Sabatier de Toulouse (FRANCE), 1989. 